University of Nebraska - Lincoln

DigitalCommons@University of Nebraska - Lincoln

$12-2019$

\title{
Behind the Scenes of Noninvasive Brain- Computer Interfaces: A Review of Electroencephalography Signals, How They Are Recorded, and Why They Matter
}

\author{
Kevin M. Pitt \\ Jonathan S. Brumberg \\ Jeremy D. Burnison \\ Jyutika Mehta \\ Juhi Kidwai
}

Follow this and additional works at: https://digitalcommons.unl.edu/specedfacpub

Part of the Special Education and Teaching Commons

This Article is brought to you for free and open access by the Department of Special Education and Communication Disorders at DigitalCommons@University of Nebraska - Lincoln. It has been accepted for inclusion in Special Education and Communication Disorders Faculty Publications by an authorized administrator of DigitalCommons@University of Nebraska - Lincoln. 


\title{
TUTORIAL
}

\section{Behind the Scenes of Noninvasive Brain- Computer Interfaces: A Review of Electroencephalography Signals, How They Are Recorded, and Why They Matter}

\author{
Kevin M. Pitt, ${ }^{1}$ Jonathan S. Brumberg, ${ }^{2}$ \\ Jeremy D. Burnison, ${ }^{3}$ Jyutika Mehta, ${ }^{4}$ \\ and Juhi Kidwai ${ }^{2}$
}

1 Department of Special Education and Communication Disorders, University of Nebraska-Lincoln, NE

2 Department of Speech-Language-Hearing: Sciences \& Disorders, University of Kansas, Lawrence, KS

3 Department of Scientific Support, Brain Vision, LLC, Morrisville, NC

4 Department of Communication Sciences \& Disorders, Texas Woman's

University, Denton, TX

Corresponding author - Kevin M. Pitt, email kevin.pitt@unl.edu

\begin{abstract}
Purpose: Brain-computer interface (BCI) techniques may provide computer access for individuals with severe physical impairments. However, the relatively hidden nature of BCI control obscures how BCI systems work behind the scenes, making it difficult to understand "how" electroencephalography (EEG) records the BCIrelated brain signals, "what" brain signals are recorded by EEG, and "why" these signals are targeted for BCI control. Furthermore, in the field of speechlanguagehearing, signals targeted for BCI application have been of primary interest to clinicians and researchers in the area of augmentative and alternative
\end{abstract}

Published in Perspectives of the ASHA Special Interest Groups 4:6 (December 2019).

doi 10.1044/2019_PERS-19-00059

Copyright (C) 2019 American Speech-Language-Hearing Association. Used by permission. Submitted August 27, 2019; accepted September 5, 2019; published 26 December 2019.

Editor for ASHA: Alison Behrman

Disclosures - The authors have no relevant financial or nonfinancial interests to disclose. 
communication (AAC). However, signals utilized for BCI control reflect sensory, cognitive, and motor processes, which are of interest to a range of related disciplines, including speech science.

Method: This tutorial was developed by a multidisciplinary team emphasizing primary and secondary BCI-AAC-related signals of interest to speech-language-hearing.

Results: An overview of BCI-AAC-related signals are provided discussing (a) "how" $\mathrm{BCI}$ signals are recorded via EEG; (b) "what" signals are targeted for noninvasive $\mathrm{BCI}$ control, including the $\mathrm{P} 300$, sensorimotor rhythms, steady-state evoked potentials, contingent negative variation, and the N4Oo; and (c) "why" these signals are targeted. During tutorial creation, attention was given to help support EEG and $\mathrm{BCI}$ understanding for those without an engineering background.

Conclusion: Tutorials highlighting how BCI-AAC signals are elicited and recorded can help increase interest and familiarity with EEG and BCI techniques and provide a framework for understanding key principles behind BCIAAC design and implementation.

$\mathrm{E}$ lectroencephalography (EEG) techniques noninvasively record brain activity at the level of the scalp via electrodes placed in a cap. The application of EEG techniques allows investigators to understand what happens in the brain when someone completes different tasks, such as those related to movement, speech perception, language processing, and cognitive processes, in addition to exploring differences in brain activity demonstrated for those with varying physical, cognitive, and/or sensory impairments. One area of rapidly expanding EEG research focuses on building technologies around the recorded brain signals with the aim of providing computer control for those with severe physical impairments who may find current methods of computer access ineffective or inefficient. Currently, all computer access methods require some form of physical movement for access (e.g., eye movement for eye gaze systems). However, brain-computer interface (BCI) technology seeks to translate the recorded brain activity into computer control, circumventing the necessity for an individual to possess a reliable form of motor movement for computer access. Consequently, BCI technology may serve as a computer access method, which allows individuals with severe physical impairments to utilize computer systems for varying applications such as augmentative and alternative communication (AAC) control (e.g., Brumberg, Pitt, Mantie-Kozlowski, \& Burnison, 2018). Therefore, in the field of speechlanguage-hearing, signals targeted for BCI application have been of primary interest to clinicians and researchers in the areas of AAC and 
assistive technology. Furthermore, the signals utilized for BCI control are applicable to a range of fields related to speech-language-hearing, including speech science, to better understand the sensory, motor, and cognitive processes.

High-technology techniques for AAC access, such as eye gaze, can sometimes be complex for individuals who use AAC to understand (McCord \& Soto, 2004). However, as existing AAC access methods require some form of physical movement, it is possible to make basic associations between an action (e.g., eye movement or switch activation) and device control. In contrast, the relatively covert or hidden nature of $\mathrm{BCI}$ control obscures how these systems work for those who are not directly involved in BCI research and signal-processing developments. Specifically, it may be unclear "how" EEG records the BCIrelated brain signals, "what" brain signals are being recorded by EEG, and "why" these signals are targeted for BCI control. Understanding the how, what, and why behind $\mathrm{BCI}$ is an important foundation for professionals looking to implement $\mathrm{BCI}$ technology and is necessary to comprehend how EEG signals are acquired and the rationales behind $\mathrm{BCI}$ designs that are tailored to elicit a specific target EEG signal such as the $\mathrm{P} 300$ for $\mathrm{BCI}$ control. Furthermore, while there are resources reviewing $\mathrm{BCI}$ techniques (e.g., Akcakaya et al., 2014; Brumberg, Pitt, Mantie- Kozlowski, et al., 2018; Wolpaw, Birbaumer, McFarland, Pfurtscheller, \& Vaughan, 2002), there are limited works available focused on educating clinical and research professionals, along with other BCI stakeholders (e.g., clients, family, and caregivers), about the preliminary processes governing how EEG signals are recorded, why a given signal is suitable for BCI use, and how this impacts interface design. These foundations may not be fully intuitive for individuals without a background in science, engineering, or BCI development, impeding the involvement of clinical professionals and stakeholders in the BCI process. This lack of background knowledge in BCI processes may decrease stakeholders' comfort and familiarity with high-technology- based AAC and BCI applications (e.g., Baxter, Enderby, Evans, \& Judge, 2012; Blain-Moraes, Schaff, Gruis, Huggins, \& Wren, 2012), possibly increasing their anxiety (Jeunet, N'Kaoua, \& Lotte, 2016) and ultimately impeding the translation of BCI research into clinical practice by limiting stakeholder involvement. Therefore, by demystifying the processes behind BCI and EEG technology (Jeunet et al., 2016), this tutorial aims to provide an EEG and BCI overview 
regarding (a) how EEG records BCIrelated signals, (b) what EEG signals are targeted for BCI control and development, and (c) why these signals are targeted.

\section{Method}

A multidisciplinary team, including a BCI-AAC developer, a neuroscientist with experience in BCI development and EEG data collection and analyses, and three speechlanguage pathologists (two with experience in the clinical translation of BCI-AAC technology and one with experience in neuroscience and EEG-based research), identified foundational principles of EEG function. In addition, the multidisciplinary team identified major paradigms for discussion that include auditory and visually elicited EEG signals primarily used for direct BCI control (i.e., auditory and visual P30o, steady-state evoked potentials, sensorimotor modulations, and the contingent negative variation [CNV]), along with a secondary signal, the N400, which to date has largely been utilized to improve P300-based BCI accuracy. Tutorial sections were informed via recent literature on EEG-based $\mathrm{BCI}$ research and experience, with an emphasis on research related to the field of speech-languagehearing and speech science. To outline the "how," "what," and "why" of BCI technology, the tutorial is split into two sections, with Section 1 discussing "how" brain signals are recorded via EEG and Section 2 discussing "what" primary and secondary signals are being recorded, in addition to "why" these signals are targeted for BCI applications.

\section{"How" EEG Signals Are Recorded}

To understand the utility of $\mathrm{BCI}$ for $\mathrm{AAC}$ applications, it is important to gain a fundamental knowledge about why EEG is used for BCI-AAC applications, along with an understanding of how the EEG system records the targeted BCI signals. Therefore, in the following section, we will outline the basic foundations necessary for understanding the use of EEG in a BCI-AAC context, including the suitability of EEG for BCIAAC applications, a description of the EEG system, and the underlying brain activity the system records. 


\section{EEG for BCI Primer}

In contrast to invasive brain recording methods such as electrocorticography, which require invasive surgery for the electrode array to be placed on or within the brain's cortex (e.g., Brumberg \& Guenther, 2010), EEG noninvasively measures brain activity at the scalp via electrodes placed in a cap, which the individual wears during EEG recordings. Therefore, while EEG provides decreased signal- to-noise ratios in comparison to invasive methods (e.g., Brumberg \& Guenther, 2010), it provides a practical alternative to record brain signals used for BCI-AAC control without requiring invasive surgery. A primary reason EEG is used for $\mathrm{BCI}$ control is due to its high temporal resolution, which allows for the measurement of brain activity from $1 \mathrm{~ms}$ to the next. As many aspects of attention and perception appear to operate on a scale of tens of milliseconds, this high temporal resolution allows a range of neurological signals to be identified in the EEG signal, which may be used for BCI-AAC control such as the P30o, steady-state evoked potentials, and those involved in motor processes (e.g., Akcakaya et al., 2014; Brumberg, Pitt, Mantie-Kozlowski, et al., 2018). These varied brain signals can be elicited via paradigms that do not require overt physical movements, making them ideal candidates for communication device control for those without functional motor movements or those who find precise movements (e.g., eye movements) highly fatiguing.

Traditionally, brain signals used in BCI-AAC applications utilize EEG systems with silver chloride or tin electrodes, which require the application of electrolyte to provide a conductive path between the scalp and the recording electrode, lowering electrical impedance. Different BCI-AAC systems utilize varied numbers of electrodes, depending on the specific BCI system and the targeted signal. Research-based BCI applications may use 64 electrode locations or more (e.g., Brumberg, Burnison, \& Pitt, 2016). However, more commercial BCI systems may use less electrode locations (e.g., eight electrode locations; Guger et al., 2009) to limit setup burdens. To understand the brain areas involved in the generation of BCI signals (e.g., visual, auditory, sensorimotor), it is important to understand the basic foundations of how electrode locations are identified. Furthermore, the electrode location of primary interest for a target BCI signal may influence BCI-AAC assessment criterion, as visually elicited $\mathrm{BCI}$ signals such as the visual 
P300 and steady-state visually evoked responses are commonly recorded on posterior recording electrodes (e.g., P, O, and PO locations; Combaz et al., 2013), which may be impeded by wheelchair headrests (e.g., Pitt \& Brumberg, 2018a). EEG electrode locations are traditionally identified using the 10-20 system (Jasper, 1958), which describes different electrode recording locations by using numbers and letters to identify the electrodes, adjacent brain areas, and lateralization. Regarding underlying brain areas, electrodes identified with the letter $\mathrm{F}$ are located over frontal areas of the brain, electrodes identified with $\mathrm{C}$ are located over central areas, electrodes identified with $\mathrm{T}$ are located over temporal areas, electrodes identified with $\mathrm{P}$ are located over parietal areas, and electrodes identified with $\mathrm{O}$ are located over occipital areas at the back of the head. Evennumbered electrode locations indicate right-side lateralization, odd numbers indicate leftside lateralization, and $\mathrm{Z}$ or zero refers to electrodes placed along the midline (e.g., Teplan, 2002). For instance, electrode locations CZ, C3, and $\mathrm{C}_{4}$ refer to the centrally located sensorimotor areas of the brain found over midline (CZ), left lateral ( $\left.\mathrm{C}_{3}\right)$, and right lateral ( 4 4) locations (for a full review of the 10-20 and other placement systems, see Jurcak, Tsuzuki, \& Dan, 2007).

Ultimately, the EEG techniques described above measure the summed electrical activity of thousands to millions of neurons. When neurons in the brain communicate, they release neurotransmitters across the space between them called the synapse. The neurotransmitter released from the presynaptic neuron then binds with receptors on the other side of the synapse, which are known as postsynaptic receptors. The released neurotransmitter that binds to these postsynaptic receptors located on the neuron's dendrites will result in either postsynaptic inhibition or excitation. An action potential will be generated with sufficient excitation propagating the signal along the axon to other neurons. The electrical activity associated with individual action potentials is not sufficient for observation using scalp EEG, but the voltages of the postsynaptic potentials of cortical pyramidal cells, when summed together during synchronous firing, become large enough to be recorded by EEG. 


\section{"What" Brain Signals Are Recorded by EEG and "Why" They Are Targeted for BCI-AAC Control}

A variety of EEG paradigms are used to elicit brain signals related to $\mathrm{BCI}$ control and development, and each targeted signal is related to different sensory, cognitive, motor, and language processes, each having its own application to BCI. The following section provides a review of primary signal used for direct BCI-AAC control (i.e., auditory and visual P300, steady-state evoked potentials, sensorimotor modulations, and the $\mathrm{CNV}$ ), along with a secondary signal, the N4Oo, which to date has largely been utilized to improve P30o-based BCI accuracy. Furthermore, to inform BCI-AAC assessment, fundamental factors influencing signal production and $\mathrm{BCI}$ performance will be noted. A diagram highlighting different brain signals and how they are utilized for BCI control is provided in Figure 1. In greater detail, Stage 1 of Figure 1 reflects the EEG recording of brain signals via the EEG electrodes placed in a fabric cap, as described in the "how" portion of this tutorial. Stage 2 reflects the second portion of this tutorial, outlining "what" primary signals are utilized for BCI control including steadystate evoked potentials (top line of the EEG output), P300 (second line of the EEG output), and sensorimotor rhythm (bottom line of the EEG output). Stage 3 then reflects the final tutorial portion, outlining "why" the primary BCI signals are targeted for communication device control (e.g., the interface item associated with the occurrence of the P300 may be identified for selection), along with examples of graphical interfaces for BCIAAC. The N4OO is not directly included in this figure since its primary role in $\mathrm{BCI}$ to date is to support increased performance for grid-based P300 BCI devices.

\section{Primary Signals Related to BCI-AAC Control}

\section{Auditory and Visual P3oo Event-Related Potential}

The P30o event-related potential (ERP) is a very popular signal targeted for BCI-AAC control due to its relatively large amplitude and ability to be elicited through auditory, visual, and tactile sensory modalities (e.g., Guger et al., 2009). ERPs are small voltage changes recorded by EEG over time, which are generated in response to specific events or stimuli (e.g., onset of a visual or auditory stimulus; Luck, 


\section{Brain-computer interface $(\mathrm{BCl})$}

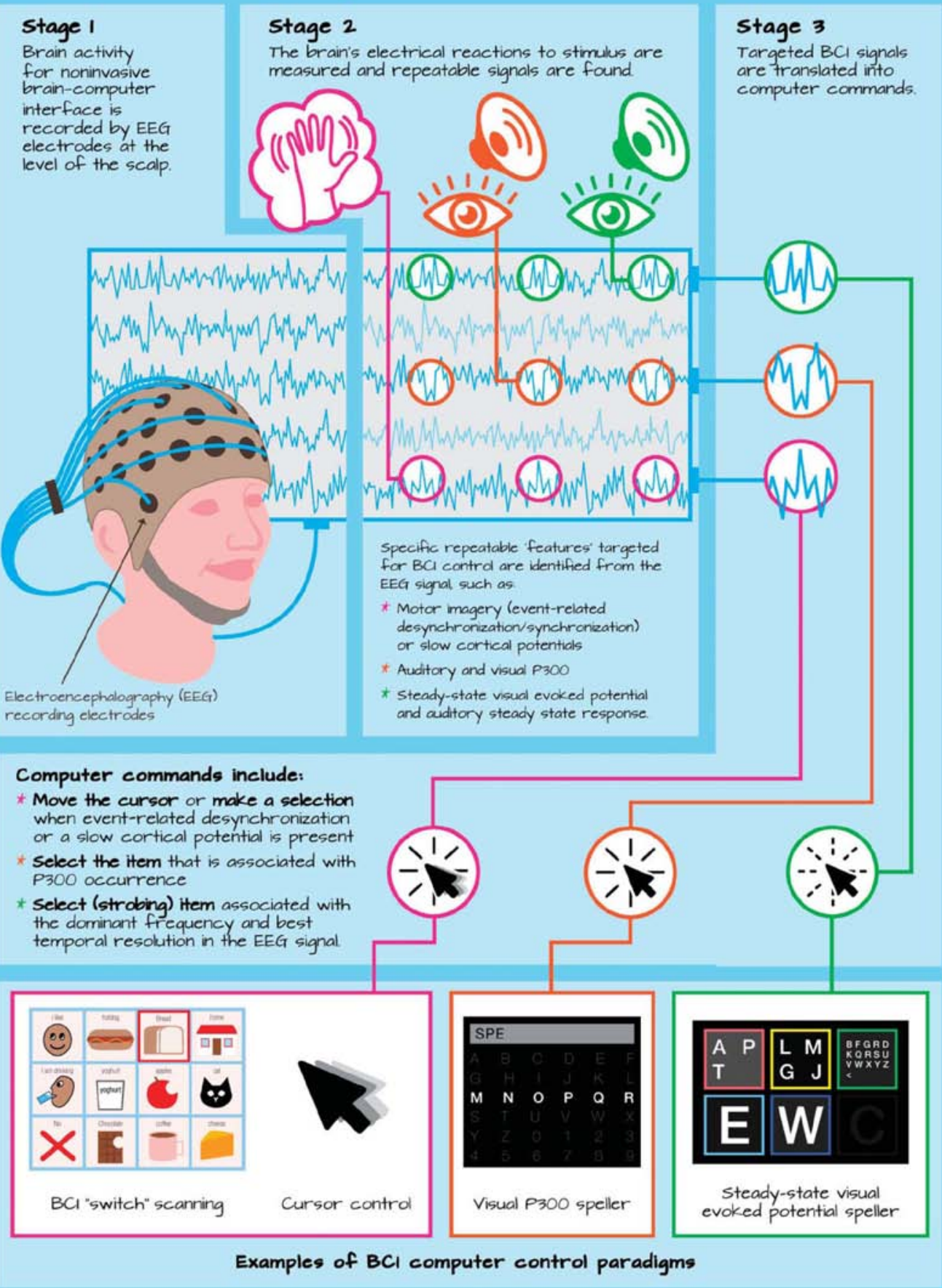

Figure 1. A schematic outlining the basic stages of brain-computer interface (BCI) operation, including "how" the BCI signal is recorded (Stage 1), "what" signals are targeted for BCI control (Stage 2), and "why" they are targeted for BCI control (Stage 3), along with examples of different BCI control paradigms. EEG = electroencephalography. 
2014). Increased ERP deflections in the EEG signal, either positive or negative, are associated with increased neural activity, with decreased latency representing a shorter time for allocation of associated cortical resources (e.g., Polich, Howard, \& Starr, 1983). As with most ERPs, the P3oo signal name reflects the polarity and timing of the EEG voltage deflection after the initial stimulus onset or event. Specifically, the $\mathrm{P}_{300}$ is a positive-going deflection in the EEG signal, reaching its peak amplitude at latency of approximately $300 \mathrm{~ms}$ (see Figure 2), though its latency may vary from around 250 to $500 \mathrm{~ms}$, depending upon factors such as an individual's age, stimulus modality (i.e., auditory, visual, and tactile presentations), and other task conditions (Polich, 2007).

The P30o ERP continues to receive a lot of attention in the EEG literature since its discovery by Sutton, Braren, Zubin, and John (1965) and is elicited through tasks that require conscious discrimination of a target stimulus. Fully, the P3oo is commonly elicited through an oddball presentation paradigm. During an oddball presentation paradigm, the individual is presented with a series of stimuli that include a frequently appearing "background" stimulus intermixed with infrequent or novel stimulus known as the "oddball" (e.g., Donchin, Ritter, \& McCallum, 1978). For instance, an individual may be required

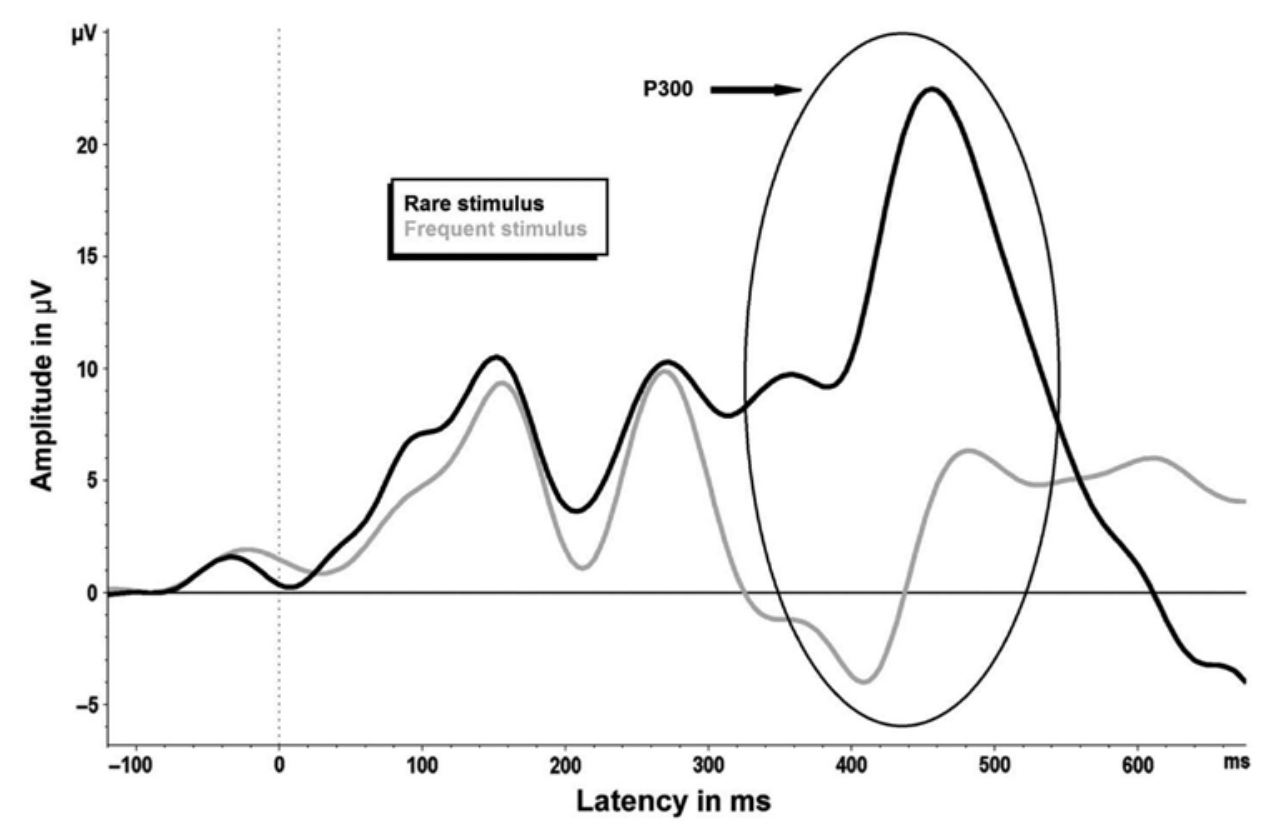

Figure 2. The $\mathrm{P} 300$ event-related potential, during which a positive-going electroencephalography deflection is noted at approximately $300 \mathrm{~ms}$. 
to listen for a less frequent high-pitched tone that occurs in a repeating train of more frequent, low-pitched, standard tones or count the number of times a target grid item is visually highlighted, among illumination of all other grid items. Through this paradigm, a P300 ERP occurs following identification of each high tone or target grid item presentation, with $\mathrm{P} 300$ amplitude increasing as the probability of identifying the target decreases (e.g., Duncan-Johnson \& Donchin, 1977). Decreasing the probability of target stimulus presentation to increase $\mathrm{P} 300$ amplitudes creates a trade-off in experimental designs, however, as fewer rare/target stimuli will be presented in a given experimental time window. Therefore, stimulus probability must be balanced with obtaining sufficient target trials for the P30o ERP to be resolved over background noise.

The neural mechanisms governing P300 production are thought to reflect information processing, the allocation of attentional resources, and memory access or encoding during context updating (e.g., Donchin \& Coles, 1988; Polich, 2007). In more detail, during the oddball paradigm, the early subcomponent of the P300 ERP (also known as the $P_{3} a$ ) is thought to reflect attention-driven processes that, along with memory access, help discriminate whether the current stimulus is different (novel) in comparison to the ones preceding it. If the stimulus is found to be different, the "full" P300 ERP is produced, due to revision of the individual's underlying mental representation and memory storage eliciting the later $\mathrm{P} 3 \mathrm{~b}$ subcomponent (e.g., Donchin, 1981; Polich, 2007). How the P300 signal specifically reflects these attention- and memory-related processes is still somewhat unclear, but the P300 may represent the inhibition of extraneous mental activity to facilitate enhancement of task-related cognitive processes (see Polich, 2007, for a review).

\section{"Why" Is the P300 Targeted for BCI Control?}

For BCI applications, the $\mathrm{P} 300$ interface is designed to elicit the P3oo via the oddball paradigm using either a serial or grid-based presentation. For example, to access a grid-based P300 BCI system (e.g., Donchin, Spencer, \& Wijesinghe, 2000), the individual decides on the communication item they wish to select before the presentation begins. The undesired items serve as the frequent stimulus, and the desired item serves as the novel/infrequent stimulus. The 
P30o display then randomly highlights all grid items (e.g., by turning them from gray to white or a color; e.g., Ikegami, Takano, Saeki, \& Kansaku, 2011), while the individual focuses their attention on the target item they wish to select (e.g., Donchin et al., 2000). When the desired item is presented, a larger $\mathrm{P} 300$ is elicited in comparison to the other stimuli, and after a few presentations of each grid item, the averaged ERPs for each stimulus are compared. The BCI then identifies the item associated with the largest P300 occurrence and selects that communication item. In contrast to the grid layout, items may also be presented in a rapid serial visual presentation format (e.g., Oken et al., 2014) where communication items are presented one at a time in a randomized fashion from a single visual field (e.g., central). The utilization of overt attention strategy where the individual focuses their gaze on the item they wish to select has been shown to increase P300-based BCI outcomes in contrast to a covert attention strategy, where an individual focus on an item in their periphery (P. Brunner et al., 2010). Therefore, the rapid serial visual presentation paradigm is ideal for individuals with oculomotor difficulties who may struggle to attend to a desired stimulus placed in a grid format (e.g., Pitt \& Brumberg, 2018a), as items can be placed according to oculomotor abilities. P300- based BCIs are designed to elicit the maximum $\mathrm{P} 300$ amplitude and the shortest latency for the stimulus of choice by manipulating fundamentals of the oddball paradigm, such as matrix size and interstimulus interval (e.g., Sellers, Krusienski, McFarland, Vaughan, \& Wolpaw, 2006) and presentation rate (McFarland, Sarnacki, Townsend, Vaughan, \& Wolpaw, 2011). The time needed to select the appropriate letter may also be reduced by utilizing language models to guide stimuli presentation (e.g., Oken et al., 2014). It is also of relevance to interface design to understand ERP amplitudes are very small (i.e., microvolts) and thus are easily obscured in the EEG signal by noise from varying sources such as electrical interference and muscle movements (e.g., Fisch, 2000; Luck, 2014). Therefore, to improve the signal-to-noise ratio, the oddball target and frequent stimulus are commonly presented on multiple occasions, with each item in the grid being flashed more than once before the BCI makes a selection. Thus, it is the amplitude and latency of the averaged ERP waveform that are used by the BCI to assess the neural P300 response. While an increased number of trials improves the quality of the P3oo recordings, the time it takes 
for the BCI to make an item selection is increased, slowing overall communication rate.

To date, visual and auditory $\mathrm{P} 300 \mathrm{BCI}$ systems have received the most attention, with visually based devices currently involved in home use by individuals with amyotrophic lateral sclerosis (ALS; e.g., Wolpaw et al., 2018), and a recent meta-analysis of BCI performance accuracies for individuals with ALS revealed the pooled accuracy of visually based P300 BCI devices across 15 studies was $72.94 \%$, with a 95\% confidence interval ranging from $64.26 \%$ to $81.62 \%$ (Marchetti \& Priftis, 2015). Furthermore, a recent longitudinal investigation by Wolpaw et al. (2018) demonstrated successful use of a visual P300 BCI system for individuals with ALS with 14 participants, from an original cohort of 39, progressing to independent at-home BCI use. Furthermore, seven of the eight remaining participants elect to keep the BCI for continued use at the end of the 18-month study period. It should be noted, however, that only five people withdrew from the study due to limitations in the BCI system or preferences for another device, with the primary reason for withdrawal being changes in health-related factors. Auditory-based P300 devices are a less frequently utilized form of $\mathrm{BCI}$ technology in comparison to their visual counterparts, and therefore, performances for individuals with neuromotor disorders are less clear. Nevertheless, initial performance may be decreased for auditorybased P3oo devices in comparison to their visual counterparts, due to difficulties with auditory attention and an increased cognitive load associated with mapping of the grid-based system into the auditory domain (e.g., translating rows and columns of the visual grid into a number system; Kübler et al., 2009). However, the effects of long-term BCI training on auditory $\mathrm{P} 300 \mathrm{BCI}$ performance requires further investigation. It is also clinically relevant to note that BCI performance accuracies for individuals with neuromotor impairments are variable for P30o devices, as with other BCI techniques, and an individual's unique profile can influence BCI outcomes (e.g., Fried-Oken, Mooney, Peters, \& Oken, 2013; Pitt \& Brumberg, 2018a). For instance, improved P300 BCI performance is associated with unimpaired selective attention and working memory skills, along with positioning factors that help ensure posterior recording electrodes are unimpeded. Therefore, similar to existing AAC procedures, an individual's unique current and future profile and environment should be considered in BCI assessment (see Pitt \& Brumberg, 2018a, for a review). 
Auditory and Visual Steady-State Evoked Potentials

Similar to the P300, steady-state evoked potentials allow for computer access via sensory stimulation. However, in contrast to the P300, which reflects voltage changes over time, steady-state evoked potential-based BCI control is achieved via evaluation of task-related frequency components in the EEG signal. More specifically, steady-state visual evoked potentials (SSVEPs) utilize rhythmic brain oscillations that are modulated by a driving visual stimulus repeating at a fixed rate, such as a flickering light or strobing icon (e.g., Regan, 1966). During SSVEP paradigms, the individual attends to a constant SSVEP stimulus, which causes synchronous neural firing that follows the presentation rate of the visual stimulus (Horwitz et al., 2017). This synchronous firing produces a robust response with stable amplitude and phase over time (Regan, 1966). The SSVEP is periodic in nature, with stimulation frequencies commonly between 8 and $30 \mathrm{~Hz}$. For example, in an SSVEP paradigm where multiple stimuli are presented simultaneously at different stimulation frequencies, the stimulation frequency that the individual is attending induces a greater magnitude of neural synchrony (Müller-Putz, Scherer, Brauneis, \& Pfurtscheller, 2005). This synchronous neural firing increases the energy present in the target frequency band, when evaluated via a time-frequency analysis, along with increasing its temporal resolution (Lin, Zhang, Wu, \& Gao, 2007) in comparison to the other nontarget stimuli over posterior recording electrodes. The different stimuli are typically presented at different locations in the visual field to allow for discrete attention to one stimulus/frequency.

Paralleling the SSVEP, the auditory steady-state response (ASSR) is an auditory evoked potential in response to periodically presented auditory stimuli such as a string of clicks or amplitude-modulated tones between 20 and $100 \mathrm{~Hz}$ (Cohen, Rickards, \& Clark, 1991; Hill \& Schölkopf, 2012). The ASSR can be localized to primary and secondary auditory cortex (Liégeois-Chauvel, Lorenzi, Trébuchon, Régis, \& Chauvel, 2004), with rhythmic brain oscillations in auditory cortex being modulated by the frequency of the driving input stimulus. 
"Why" Are Visual and Auditory Steady-State Evoked Potentials Targeted for BCI Control?

While BCI applications of the ASSR are still in the early stages, SSVEP has an established history for BCI application due to its high signal-tonoise ratio (Srinivasan, Bibi, \& Nunez, 2006). For example, in a fourchoice SSVEP display, Item 1 may flicker at $8 \mathrm{~Hz}$, Item 2 may flicker at $9 \mathrm{~Hz}$, Item 3 may flicker at $10 \mathrm{~Hz}$, and Item 4 may flicker at $11 \mathrm{~Hz}$. In this SSVEP paradigm, the frequency of the item associated with the target demonstrates increased neural synchrony and temporal correlation in the EEG signal in comparison to the nontarget items. Therefore, the BCI decoding algorithm selects the target item by identifying the stimuli associated with the largest magnitude of response during the trial. With the SSVEP Shuffle Speller (Higger et al., 2017), the individual selects different boxes of letters, each flickering at their own specific frequency. Through a language model, selections are made until the individual has only one letter left to select. Furthermore, SSVEP-based BCIs can allow access to graphical interfaces presenting a large array of items for selection, such as a full keyboard layout with 30 selection options (e.g., Hwang et al., 2012). In the full keyboard paradigm, each letter and symbol flickers at a slightly different rate (e.g., letter A flickers at $7 \mathrm{~Hz}, \mathrm{~B}$ flickers at $7.8 \mathrm{~Hz}, \mathrm{Z}$ flickers at $6 \mathrm{~Hz}$ ), with the most similar stimulation frequencies (e.g., 7 and $7.1 \mathrm{~Hz}$ ) being spread across the keyboard display to avoid overlap of adjacent stimulus frequencies.

For ASSR-based BCIs, individuals are instructed to attend to a specific frequency-tagged sound stream coming from a specific location (Kim et al., 2011). Similar to the SSVEP, the sound stream to which the individual is attending can be identified by the BCI algorithm through increased ASSR frequency amplitude, resulting in item selection. For instance, Hill et al. (2014) investigated ASSR-based BCI paradigm for making yes and no selections, utilizing auditory attention to one of two amplitude-modulated "beeping" sound streams of $768 \mathrm{~Hz}$ to the right ear and $512 \mathrm{~Hz}$ to the left ear.

Recent studies are beginning to support the feasibility of SSVEP use by individuals with neuromotor disorders (e.g., Hwang et al., 2017), with reported performance accuracies such as $\geq 70 \%$ (Combaz et al., 2013) and 76.99\% (Hwang et al., 2017). However, SSVEP-based $\mathrm{BCI}$ performance is variable (e.g., 18.75\%-73\% for individuals with 
various neuromotor disorders; Brumberg, Nguyen, Pitt, \& Lorenz, 2019) and is influenced by an individual's unique profile. Similar to P30o-based BCIs, SSVEP performance is supported by factors such as individuals' oculomotor strengths, which allows for utilization of overt attention strategies (Brumberg et al., 2019; Peters et al., 2018). However, SSVEP items may be arranged to support oculomotor strengths to improve BCI accuracy (e.g., Brumberg et al., 2019). Furthermore, positioning factors, such as pressure from a wheelchair headrest, and uncontrolled neck and muscle movements may impede posterior electrode recordings, which decreases SSVEP BCI performance below the aforementioned levels (Daly et al., 2013). Finally, in addition to cognitive factors such as attention, due to the rapidly flickering stimuli, an individual's history of seizures is an important consideration when considering SSVEP-based BCI use (e.g., Pitt \& Brumberg, 2018b). In comparison to SSVEP, ASSR devices are an emerging BCI technique. Therefore, the utility of ASSR-based BCIs for individuals with neuromotor disorders is currently unclear but under investigation.

\section{Sensorimotor Modulations}

Similar to the steady-state evoked potentials such as the SSVEP, sensorimotor-based BCI control is obtained through time-frequency analysis of the energy levels present in different frequency bands. However, in comparison to influencing the synchrony of neural oscillation through external sensory stimulation (e.g., a flickering stimuli), sensorimotor oscillations are modulated by tasks such as physical or imagined movements, during which an individual mentally recreates an action without physical execution. Brain oscillations or rhythms refer to the repetitive, synchronous, electrical activity generated by neurons. When the brain is relaxed or at rest, it is described as being in an idling state, and in this state, a large number of neurons produce synchronized rhythmic activity between approximately 8 and $12 \mathrm{~Hz}$ to possibly govern inhibitory and excitatory cortical processes to manage energy use (e.g., Neuper \& Pfurtscheller, 2001; Pfurtscheller, Brunner, Schlögl, \& Lopes da Silva, 2006; Pfurtscheller \& Lopes da Silva, 1999; Pfurtscheller \& Neuper, 2010). However, when an individual becomes engaged in processing cognitive, sensory, and/or motor-based information, this neural synchrony between approximately 8 and 12 $\mathrm{Hz}$ decreases, as neurons begin firing at different rates to accomplish 
the given task. Therefore, tasks such as a physical or imagined movement and cognitive tasks such as word association and arithmetic (e.g., mental subtraction; Friedrich, Scherer, \& Neuper, 2012) may be used to modulate the energy levels (i.e., power) of different frequency bands within the EEG signal, including alpha (approximately 8-12 Hz), which is called mu when measured over sensorimotor areas (Kuhlman, 1978); beta (approximately 18-26 Hz); and gamma (> $35 \mathrm{~Hz}$ ). In more detail, each rhythm is identified by its own scalp location and frequency range, and when the brain is relaxed or at rest, synchronous neural firing causes an increase in alpha band power known as event-related synchronization (see Figure 3). However, a time-frequency analysis reveals that, when neuronal activity becomes desynchronized due to task-related engagement of cortical areas, a decrease in alpha band power is noted in the EEG signal. When this neuronal desynchronization occurs following an event (e.g., presentation of an external stimulus prompting motor [imagery] task performance), it is known as eventrelated desynchronization (Pfurtscheller \& Lopes da Silva, 1999; see Figure 3).

The focus of sensorimotor-based BCI research has been on providing $\mathrm{BCI}$ control via imagined actions, as imagined movements engage primary sensorimotor areas of the brain associated with

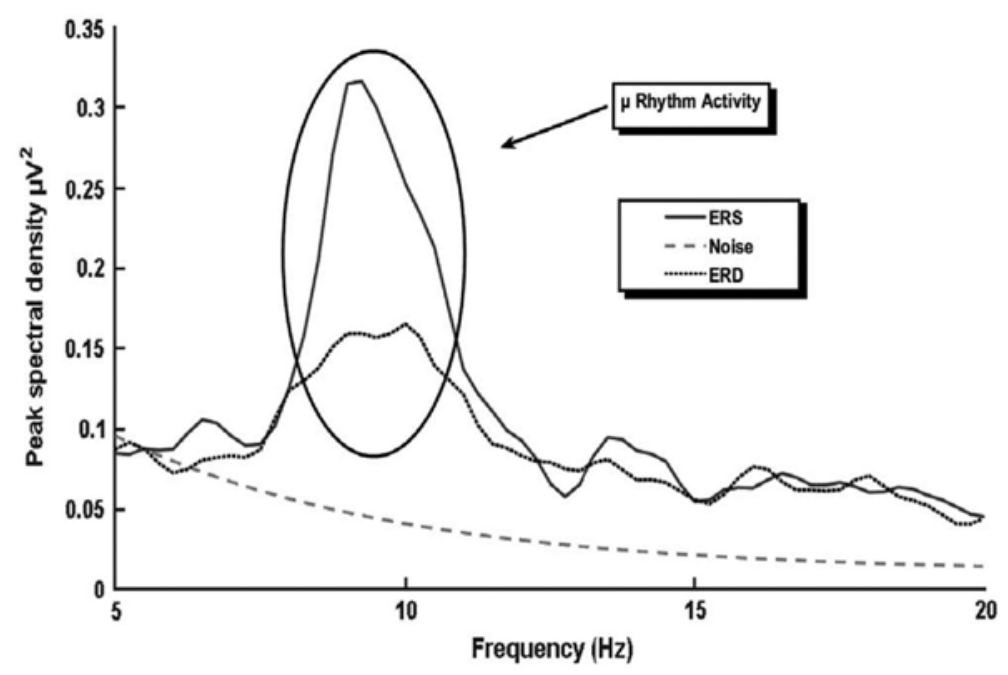

Figure 3. The sensorimotor rhythm recoded over the right hemisphere (electrode location $\mathrm{C}_{4}$ ) during imagined movement of the left hand. The larger peak denotes increased mu band power at rest (eventrelated synchronization [ERS]), whereas the lower peak demonstrates event-related desynchronization (ERD) during imagined task performance. 
neuromuscular function in a manner similar to physical movement (e.g., Neuper, Scherer, Reiner, \& Pfurtscheller, 2005; Pfurtscheller \& Lopes da Silva, 1999). However, a variety of tasks may potentially be utilized to produce general alpha desynchronization, including cognitive tasks such as word association and mental subtraction (Friedrich et al., 2012; Scherer et al., 2015). Regarding the performance of physical and imagined movements, as the nervous systems is organized to provide contralateral motor control (i.e., a right-hand movement is controlled by hand motor areas in the left hemisphere), electrodes over left sensorimotor cortex (e.g., C3) show increased desynchronization for the right-hand tasks; and right-sided electrodes (e.g., C4), for the left-hand tasks. However, for tasks where motor control areas are located closer to the brain's midline (e.g., foot motor areas), a more central sensorimotor modulation is noted (e.g., Pfurtscheller et al., 2006). Sensorimotor desynchronization associated with task performance does not occur in isolation, however, and is accompanied by synchronization of cortical areas that are not directly involved in task completion (Suffcynski, Pijn, Pfurtscheller, \& Lopes da Silva, 1999). For example, during the right-hand motor imagery, an ipsilateral synchronization (event-related synchronization) is present in the EEG signal in conjunction with the contralateral desynchronization (e.g., Pfurtscheller \& Neuper, 1997). Furthermore, during feet or tongue imagery, mu-band power increases over hand motor areas (Pfurtscheller et al., 2006).

\section{“Why” Are Sensorimotor Modulations Targeted for BCI Control?}

For some individuals, the sensorimotor rhythm may not be recordable via EEG (e.g., Blankertz et al., 2010), possibly due to anatomical variability in the shape and position of motor cortex (e.g., Morash, Bai, Furlani, Lin, \& Hallet, 2008). However, when present, since changes in the sensorimotor rhythms during performance of imagined movements parallel that of physical execution, they are a viable target to provide $\mathrm{BCI}$ control as no physical motor skills are required for sensorimotor-based access to communication. In a BCI context, changes in the sensorimotor rhythm can be detected by the $\mathrm{BCI}$ during a single trial containing physical or imagined movements and translated into computer control to access a range of BCI paradigms. These paradigms are not reliant upon visual presentation 
paradigms that incorporate flashing stimuli such as the $\mathrm{P} 300$ and SSVEP. For instance, neuronal desynchronization can be detected by the BCI when an individual imagines performing an action following highlighting of a communication item they want to select. The presence of this event-related desynchronization can trigger the BCI to make a selection, similar to switch access during scanningbased AAC paradigms (Friedrich et al., 2008; Scherer et al., 2015). Furthermore, when prompted, an individual can imagine performing a specific action (e.g., imagined rightor left-hand movements) to select different groups of letters until a single item remains for selection (e.g., Obermaier, Muller, \& Pfurtscheller, 2003). Changes in the sensorimotor rhythm can also be interpreted by the BCI in real time to provide cursor-like computer control (e.g., Wolpaw \& McFarland, 2004) in which imagining different movements results in changes in cursor position. This cursor control method could therefore allow access to a range of paradigms including selection of letters or words placed in different onscreen locations (e.g., Kübler et al., 2005; Vaughan et al., 2006). Extending the idea of cursor control to speech processing, cursor-based BCI paradigms may be used to provide real-time control of a two-dimensional formant speech synthesizer (Brumberg \& Pitt, 2019; Brumberg, Pitt, \& Burnison, 2018). It should also be considered that, in addition to motor actions and imagery, different tasks such as mental subtraction, word association, and mental rotation can create levels of desynchronization detectable by the EEG and may therefore have utility for BCI control. For example, while further research is still needed, a task other than motor imagery may yield BCI success for an individual with a lesion impairing motor cortex following stroke (see Friedrich et al., 2012, for a review).

Pooled sensorimotor BCI accuracies are reported as 70.04\% across four studies, with a 95\% confidence interval ranging from $52.22 \%$ to $87.85 \%$. However, these results need to be interpreted in light of participant heterogeneity and differences in BCI design across studies (Marchetti \& Priftis, 2015). Furthermore, while initial performances for sensorimotor BCIs utilizing auditory feedback may be associated with decreased initial BCI performances, with training, they may begin to approximate the performance levels of their visual counterparts (Nijboer et al., 2008). Regarding individual profile variability, Kasahara et al. (2012) found the desynchronization was dampened 
for individuals with ALS, especially for those with increased bulbar involvement. Therefore, the magnitude of the desynchronization used for BCI control may not be solely dependent upon the number or activation of surviving neural cells but by additional factors such as individuals' ability to recall a motor action from memory, level of fatigue, and ability to concentrate. Therefore, in addition to neurophysiological measures such as amplitude of the sensorimotor rhythm (Blankertz et al., 2010), sensorimotor BCI performance may be impacted by a range of cognitive (e.g., attention; Geronimo, Simmons, \& Schiff, 2016), psychological (e.g., motivation and confidence levels; Nijboer, Birbaumer, \& Kübler, 2010), and motor imagery-related (e.g., Neuper et al., 2005) factors. However, additional research is needed to identify the effects of longitudinal training on sensorimotor BCI performance (e.g., Daly et al., 2014).

\section{The $C N V$}

Similar to the sensorimotor rhythms, physical motor abilities are not required for an individual to learn voluntary control of slow cortical potentials, such as the CNV and Bereitschaftspotential, making them suitable for BCI application. In the context of BCI, voluntary control of slow cortical potentials can be learned through feedback in operant conditioning paradigms (e.g., the position of an onscreen cursor changes in relation to slow cortical potential amplitudes; Neumann \& Birbaumer, 2003). The CNV is a slow cortical potential ( $<1 \mathrm{~Hz}$; e.g., Brumberg, Pitt, Mantie-Kozlowski, et al., 2018) that was first described by Walter, Cooper, Aldridge, McCallum, and Winter (1964) and is characterized as an EEG signal with a negativegoing amplitude associated with one's degree of cortical arousal during attentional anticipation, response preparation, and information processing (e.g., Nagai et al., 2004; Segalowitz \& Davies, 2004; see Figure 4). The CNV contains both cognitive and motor components and is commonly elicited during go/no-go response paradigms during which the CNV increases in negativity between a warning cue (Stimulus 1) and an imperative stimulus (Stimulus 2), which prompts the participant to complete an action (e.g., to press a button). For instance, during a typical CNV paradigm, the first stimulus will inform the participant if they will or will not perform a set action when the second stimulus is presented. During trials 


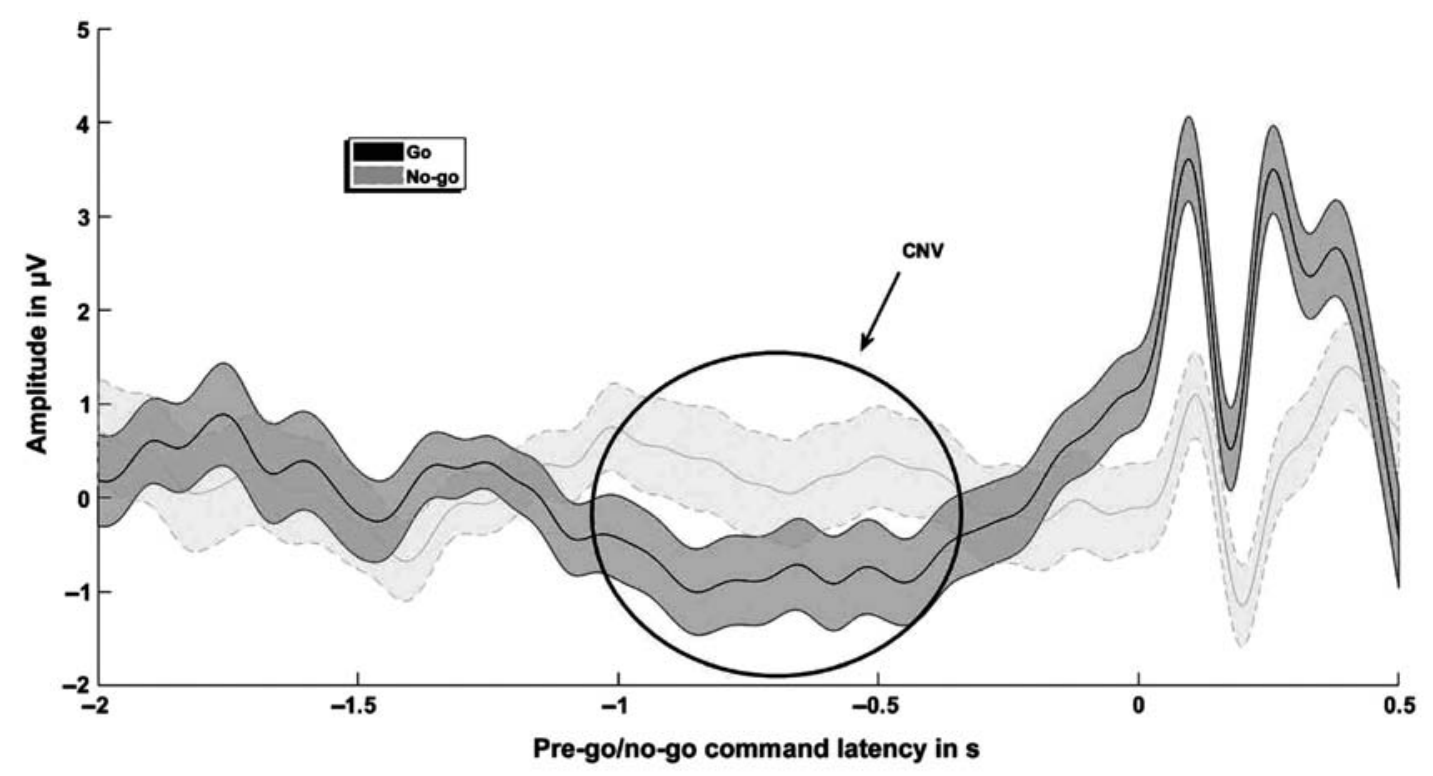

Figure 4. The contingent negative variation (CNV), showing a negative-going electroencephalography deflection prior to task onset.

where the Stimulus 1 prompts the participant to prepare to perform the action upon presentation of Stimulus 2 (known as go trials), an increasing negative drift is present in the EEG signal prior to task onset in comparison to "no-go" trials where Stimulus 1 prompts the participant to remain at rest for the trial duration (e.g., Taylor, Gavin, \& Davies, 2016). However, Stimulus 1 may also serve as a general "prepare for action" prompt, with Stimulus 2 instructing the participant to go or not to go. During response preparation between Stimuli 1 and 2, the $\mathrm{CNV}$ may be divided into two phases, an early orienting phase known as the $O$-wave and a later expectancy and preparation phase known as the E-wave (e.g., J. F. Brunner et al., 2015; Taylor et al., 2016), both of which are influenced by cognitive and motor factors (e.g., Lukhanina, Karaban, Burenok, Mel'nik, \& Berezetskaya, 2006). Specifically, the Owave is greatest at midline frontal electrodes and is associated with arousal and processing of stimulus characteristics such as intensity (e.g., Nagai et al., 2004), in addition to cognitive processes associated with categorical judgment (e.g., to "go" or "no-go"; Cui et al., 200o), and task maintenance and rehearsal (J. F. Brunner et al., 2015). In contrast, the late E-wave is associated with task setting (i.e., planning how to respond to the second stimulus) and the degree of sustained attentional efforts (J. F. Brunner et al., 2015; Cui et al., 2000). Furthermore, 
the late $\mathrm{CNV}$ is thought to reflect motor preparation to a greater degree than the early stage (Cui et al., 2000; Nagai et al., 2004), being influenced by motor factors, such as task complexity (Cui et al., 2000). The CNV shares a close relationship with another negative-going EEG potential indicting motor "readiness," known as the Bereitschaftspotential (Deecke, Grözinger, \& Kornhuber, 1976). The Bereitschaftspotential is commonly studied during selfinitiated movements (e.g., finger flexion and extension), which the individual performs at their own pace without external cues (e.g., Cui et al., 2000). Conversely, the CNV is time-locked to stimulus presentations, requiring increased levels of attention (Nagai et al., 2004).

\section{“Why" Is the CNV Targeted for BCI Control?}

Traditionally, cortical arousal associated with slow cortical potentials such as the CNV or Bereitschaftspotential is used for control of operant conditioning-based BCI devices (Neumann \& Birbaumer, 2003). To control operant conditioning-based BCIs such as the thought translation device (Birbaumer et al., 2000; Kübler et al., 1999), the individual learns to voluntarily control their slow cortical potentials through feedback (e.g., of cursor movement). For example, in comparison to a baseline period, a negative-going slow cortical potential amplitude during a single trial of active BCI control (reflecting increased cortical excitation) may move a cursor up, whereas a positive slow cortical potential amplitude (reflecting decreased cortical arousal) may move the cursor down (e.g., Kübler et al., 1999; Wolpaw \& Boulay, 2009). Similar to sensorimotor-based BCIs, this cursor control mechanism may be used for communication output or environmental control (Kübler et al., 1999; Neumann \& Birbaumer, 2003). Furthermore, paralleling sensorimotor-based BCIs, mastery of slow cortical potential devices may take extended training times (e.g., Neumann \& Birbaumer, 2003). Finally, while research is still in the early stages, the CNV may serve to provide switch-based access to commercial AAC scanning paradigms in which CNV occurrence prior to performance of an imagined movement triggering a BCI "switch" selection of the currently highlighted icon during item scanning (Brumberg et al., 2016).

Across six studies, the pooled classification accuracy of individuals with ALS for slow cortical potential-based BCI control was 72.94\%, 
with a 95\% confidence interval ranging from $67.32 \%$ to $83.36 \%$ (Marchetti \& Priftis, 2015). Furthermore, single-session BCI performance for an individual with advanced ALS was 62.2\% when utilizing CNVbased access to a commercial AAC display (Brumberg et al., 2016). Variability in BCI performance for slow cortical potential-based BCIs may be due in part to individual differences in CNV manifestation. For instance, for individuals with mild spastic cerebral, the late CNV may be relatively preserved (Hakkarainen, Pirilä, Kaartinen, \& Meere, 2012). However, individuals with spinal amyotrophic lateral sclerosis may present with CNV amplitudes that are increased (Hanagasi et al., 2002) or similar to neurotypical peers (Mannarelli et al., 2014), while individuals with bulbar amyotrophic lateral sclerosis may be more likely to present with decreased CNV amplitudes possibly due to cognitive impairments (Mannarelli et al., 2014).

\section{Secondary Signal Related to BCI Control}

\section{The N4OO ERP}

As many studies evaluating the N400 ERP (see Figure 5) are primarily language based, it may be surprising to see it discussed in the context of BCI. However, the N400 can be elicited via pictures and is

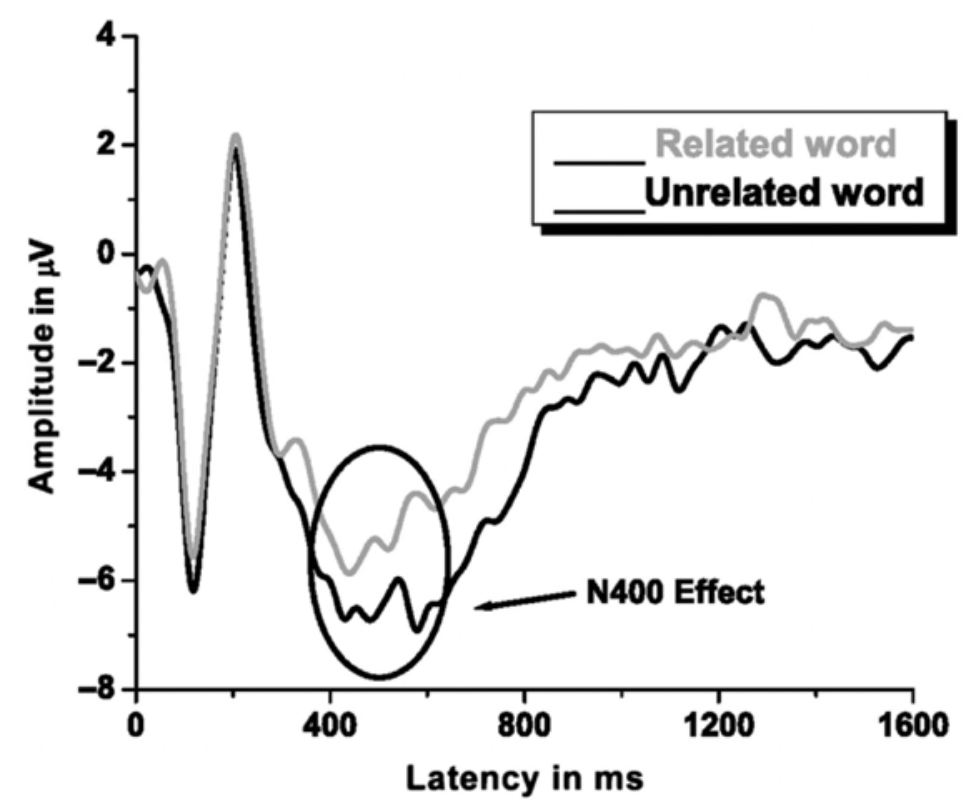

Figure 5. The N40o event-related potential, characterized by a negative-going electroencephalography deflection, is noted at approximately $400 \mathrm{~ms}$. 
sensitive to congruency and processing efforts, which may be useful in a BCI application. The N400 was originally characterized by Marta Kutas and Steven Hillyard in 1980 as a reaction to an unexpected and/or inappropriate word at the end of a sentence. Kutas and Hillyard's (1980a, 1980b, 1980c, 1982, 1983, 1984) initial series of experiments studied sentences that were grammatically correct with valid word endings, which were congruent control sentences such as "I shaved off my mustache and beard," in comparison to experimental sentences such as "I shaved off my mustache and eyebrows" (congruent and valid but low probability), "I shaved off my mustache and city" (semantically anomalous), "I shaved off my mustache and [a pictorial representation]" (novel, uninterpretable), "I shaved off my mustache and BEARD" (congruent but physically unexpected with capital letters), "I shaved off my mustache and [line drawing of a beard]" (also congruent but physically unexpected), and "I shaved off my mustache and [line drawing of a city]" (semantically anomalous and physically unexpected; examples cited from Kutas \& Federmeier, 2009). The N400 waveform is thus defined as a slow, negative deflection below the prestimulus baseline, occurring anywhere between 200 and $600 \mathrm{~ms}$ and typically peaking around $400 \mathrm{~ms}$. The amplitude component of the $\mathrm{N} 400$ is more sensitive to stimulus change compared to its latency. The variation in N40O amplitude is called N4Oo effect, and a larger N400 amplitude is expected to semantically incongruent versus semantically congruent stimuli. This increase in N400 amplitude reflects the greater neural resources needed to process the incongruent stimulus (Kutas \& Federmeier, 2011).

Since Kutas and Hillyard's initial study, the N400 is shown to be sensitive to varied manipulations, including cloze probability (the number of possible sentence endings), sentence and discourse congruity, repetition, semantic priming, lexical association, concreteness and semantic richness, word frequency, orthographic neighborhood size, and several more. The N400 is especially sensitive to semantic processing, and several linguistic and psycholinguistic accounts of how semantic context influences the N400 component during word processing have been proposed. For instance, Plante, Petten, and Senkfor (2000) have shown that the N400 amplitude is smaller if the eliciting word is semantically related rather than unrelated to the preceding word, in both visual and auditory modalities. However, Neville, Coffey, Holcomb, and Tallal (1993) suggest that N400 amplitudes may vary 
depending upon the target word position within the sentence. Essentially, they showed that words earlier in the sentence elicited a larger N400 than later words because the later words can possibly benefit from the preceding context. N400 effects are seen in ERP components following the presentation of auditory and visual stimuli as well as by signs in American Sign Language (Neville, Coffey, Lawson, Fischer, \& Emmorey, 1997). Thus, it appears that the N400 is relatively independent of the sensory modality of the linguistic input. Overall, the current literature suggests that $\mathrm{N} 400$ reflects semantic/ lexical processing of a given linguistic stimulus and that the priming effects can be interpreted as evidence of variance or modulation in semantic processing (Mehta \& Jerger, 2014).

The N40o has also been elicited in studies associated with attention (Mehta, Jerger, Jerger, \& Martin, 2009), and the N40o has utility for measuring the amount of cognitive load required for an individual during semantic memory retrieval. This is because the ability to process the information from target stimuli is highly dependent on one's ability to recall previous relevant information from any of the multimodal channels such as images or sounds. This difficulty, or cognitive load, is associated with memory representations and cues from previous content priming the meaningful probe stimulus (Federmeier \& Kutas, 2001; Lau, Phillips, \& Poeppel, 2008; Van Petten \& Luka, 2006). Therefore, when a difficult stimulus requires more effort to process, thus having more cognitive load, the N400's amplitude deflection is larger than when it is easy.

\section{"Why" Is the N40o Targeted for BCI Control?}

As the $\mathrm{N} 400$ is primarily produced by incongruency, in the absence of a motor response, future research may wish to explore the N400 and incongruency-based paradigms as the foundation for gaze-independent audiovisual BCI systems (e.g., Xie et al., 2018). However, currently in the field of $\mathrm{BCI}$, the $\mathrm{N} 400$ is largely discussed in the context of supporting improved outcomes for P300-based BCI devices, and N400 elicitation directly influences how the visual displays for gridbased P300 BCI devices are designed. Specifically, improved P300 BCI accuracy has focused on elicitation of the N400 ERP, alongside the P300, and elicitation of the N400 may contribute to improved 
P30o-based BCI accuracies even if it possibly cancels out some of the P30o ERP activity (Kaufmann, Schulz, Grünzinger, \& Kübler, 2011). The N400 is commonly elicited through the P300 "face flash" paradigm during which all items within the $\mathrm{P} 300$ grid are randomly highlighted during oddball presentation by toggling between a picture of a face and the letter. In the face flash paradigm, the N40o may reflect similar components to the classical N400, as nonlinguistic information can also elicit N400 activity through access of semantic memory (Eimer, 2000). However, whether the N400 in the face flash paradigm reflects face-specific semantic memory processes and attentional factors involved in face identification (Eimer, 2000) or reflects more general responses to stimuli processing and $\mathrm{P} 300$ paradigm characteristics is unclear (e.g., Kellicut-Jones \& Sellers, 2018). Therefore, while the N400 is not currently a common signal that is directly decoded for $\mathrm{BCI}$ performance, individuals involved in the BCI-AAC process should remain aware of its utility and impact on P300-based BCI design. Further research is warranted to fully understand the role of the N40o in P3oo paradigms for various populations.

\section{Discussion and Conclusion}

Noninvasive EEG has high temporal resolution and, without the need for invasive surgery, can be used to record brain signals in the absence of physical movement. Therefore, EEG methods are a viable option in recording brain activity underlying BCI-based access to communication. However, the process of how EEG captures the brain activity and how this recorded neural activity is translated into BCI control can be opaque for those not involved in BCI research. A lack of understanding behind basic principles governing how BCIs function may decrease the comfort of stakeholders in implementing and using BCI technology to access communication. Therefore, this tutorial provided the basic foundations regarding how EEG signals are recorded, popular EEG signals targeted for BCI development, and how these EEG signals are utilized for BCI applications to help facilitate interest and familiarity of EEG-based BCI-AAC techniques for a range of individuals and ultimately support the translation of BCIAAC into clinical practice. Based upon the information provided in this tutorial, it is clear 
that BCIs do not read an individual's "thoughts" but instead translate brain activity related to cognitive-sensory-motor processes into device control. This translation process is similar to existing AAC methods such as eye gaze, where a nonspeech task (e.g., eye movements and fixations) is translated into item selection. A review of current levels of BCI performance was also provided to help demonstrate recent advances in the field of BCI. However, while future work must continue to focus on the development of new decoding algorithms to increase BCI performances above those outlined in this tutorial, along with identifying how to decrease the high levels of workload associated with BCI use (e.g., Koch Fager, Fried-Oken, Jakobs, \& Beukelman, 2019), it is important for researchers to remain aware of how BCI will ultimately be implemented in the clinical setting (Pitt, Brumberg, \& Pitt, 2019), along with procedures providing at-home and caregiver support (e.g., Wolpaw et al., 2018). Thus, to help account for possible $\mathrm{BCI}$ performance variations and highlight how BCI is not a "one size fits all method," cognitive-sensory-motor and medical (e.g., history of seizures) factors influencing BCI outcomes were also described. However, while the foundations are laid for considering BCI in the context of existing clinical procedures such as feature matching (e.g., Pitt \& Brumberg, 2018a), much of the clinical groundwork for personalized BCI intervention has not been established, and further multidisciplinary research is needed to develop clinical guidelines for BCI intervention alongside existing AAC access methods (Pitt et al., 2019). Therefore, it is hoped that future work may build upon this tutorial to enhance multidisciplinary involvement in BCI-AAC by helping overcome procedural and language barriers between disciplines in efforts to ensure that BCI-AAC devices are developed and implemented with a person-centered focus.

Acknowledgments - This work was supported in part by the Texas Woman's University Woodcock Institute Research Grant, the Nebraska Tobacco Settlement Biomedical Research Development Fund, and the National Institutes of Health (NIDCD Ro1-DC016343 awarded to Jonathan Brumberg). The authors would like to thank Chavis Lickvar-Armstrong and Adrienne Pitt for input on the development of this tutorial and Anthony Pitt for creation of the brain-computer interface schematic in Figure 1. 


\section{References}

Akcakaya, M., Peters, B., Moghadamfalahi, M., Mooney, A. R., Orhan, U., Oken, B., ... Fried-Oken, M. (2014). Noninvasive brain-computer interfaces for augmentative and alternative communication. IEEE Reviews in Biomedical Engineering, 7, 31-49.

Baxter, S., Enderby, P., Evans, P., \& Judge, S. (2012). Barriers and facilitators to the use of high-technology augmentative and alternative communication devices: A systematic review and qualitative synthesis. International Journal of Language \& Communication Disorders, 47(2), 115-129.

Birbaumer, N., Kubler, A., Ghanayim, N., Hinterberger, T., Perelmouter, J., Kaiser, J., ... Flor, H. (2000). The thought translation device (TTD) for completely paralyzed patients. IEEE Transactions on Rehabilitation Engineering, 8(2), 190-193.

Blain-Moraes, S., Schaff, R., Gruis, K. L., Huggins, J. E., \& Wren, P. A. (2012). Barriers to and mediators of brain-computer interface user acceptance: Focus group findings. Ergonomics, 55(5), 516-525.

Blankertz, B., Sannelli, C., Halder, S., Hammer, E. M., Kübler, A., Müller, K. R., ... Dickhaus, T. (2010). Neurophysiological predictor of SMR-based BCI performance. NeuroImage, 51(4), 1303-1309.

Brumberg, J. S., Burnison, J. D., \& Pitt, K. M. (2016). Using motor imagery to control brain-computer interfaces for communication. In D. D. Schmorrow \& C. M. Fidopiastis (Eds.), Foundations of augmented cognition: Neuroergonomics and operational neuroscience: 1oth International Conference (pp. 14-25). Cham, Switzerland: Springer.

Brumberg, J. S., \& Guenther, F. H. (2010). Development of speech prostheses: Current status and recent advances. Expert Review of Medical Devices, 7(5), 667-679.

Brumberg, J. S., Nguyen, A., Pitt, K. M., \& Lorenz, S. D. (2019). Examining sensory ability, feature matching and assessmentbased adaptation for a braincomputer interface using the steady-state visually evoked potential. Disability and Rehabilitation: Assistive Technology, 14(3), 241-249.

Brumberg, J. S., \& Pitt, K. M. (2019). Motor induced suppression of the N100 ERP during motor-imagery control of a speech synthesizer brain-computer interface. Journal of Speech, Language, and Hearing Research, 62, 2133-2140.

Brumberg, J. S., Pitt, K.M., \& Burnison, J. D. (2018). A noninvasive brain-computer interface for real-time speech synthesis: The importance of multimodal feedback. IEEE Transactions on Neural Systems and Rehabilitation Engineering, 26(4), 874-881.

Brumberg, J. S., Pitt, K. M., Mantie-Kozlowski, A., \& Burnison, J. D. (2018). Braincomputer interfaces for augmentative and alternative communication: A tutorial. American Journal of Speech-Language Pathology, 27(1), 1-12.

Brunner, P., Joshi, S., Briskin, S., Wolpaw, J. R., Bischof, H., \& Schalk, G. (2010). Does the 'P30o'speller depend on eye gaze? Journal of Neural Engineering, 7(5), 056013 . 
Brunner, J. F., Olsen, A., Aasen, I. E., Løhaugen, G. C., Håberg, A. K., \& Kropotov, J. (2015). Neuropsychological parameters indexing executive processes are associated with independent components of ERPs. Neuropsychologia, 66, 144-156.

Cohen, L. T., Rickards, F. W., \& Clark, G. M. (1991). A comparison of steady state evoked potentials to modulated tones in awake and sleeping humans. The Journal of the Acoustical Society of America, 9o(5), 2467-2479.

Combaz, A., Chatelle, C., Robben, A., Vanhoof, G., Goeleven, A., Thijs, V., ... Laureys, S. (2013). A comparison of two spelling brain-computer interfaces based on visual P3 and SSVEP in locked-in syndrome. PLOS ONE, 8(9), e73691.

Cui, R. Q., Egkher, A., Huter, D., Lang, W., Lindinger, G., \& Deecke, L. (2000). High resolution spatiotemporal analysis of the contingent negative variation in simple or complex motor tasks and a non-motor task. Clinical Neurophysiology, 111(10), 1847-1859.

Daly, I., Billinger, M., Laparra-Hernández, J., Aloise, F., García, M. L., Faller, J., ... Müller-Putz, G. (2013). On the control of brain-computer interfaces by users with cerebral palsy. Clinical Neurophysiology, 124(9), 1787-1797.

Daly, I., Faller, J., Scherer, R., Sweeney-Reed, C. M., Nasuto, S. J., Billinger, M., \& Müller-Putz, G. R. (2014). Exploration of the neural correlates of cerebral palsy for sensorimotor BCI control. Frontiers in Neuroengineering, 7, 20.

Deecke, L., Grözinger, B., \& Kornhuber, H. H. (1976). Voluntary finger movement in man: Cerebral potentials and theory. Biological Cybernetics, 23(2), 99-119.

Donchin, E. (1981). Surprise! ... Surprise? Psychophysiology, 18(5), 493-513.

Donchin, E., \& Coles, M. G. H. (1988). Is the P30o component a manifestation of context updating? Behavioral and Brain Sciences, 11(3), 357-374.

Donchin, E., Ritter, W., \& McCallum, K. W. C. (1978). Cognitive psychophysiology: The endogenous components of the ERP. In E. Callaway, P. Tueting, \& S. Koslow (Eds.), Event-related brain potentials in man (pp. 349-441). New York, NY: Academic Press.

Donchin, E., Spencer, K. M., \& Wijesinghe, R. (2000). The mental prosthesis: Assessing the speed of a P30o-based brain-computer interface. IEEE Transactions on Rehabilitation Engineering, 8(2), 174-179.

Duncan-Johnson, C. C., \& Donchin, E. (1977). On quantifying surprise: The variation of event-related potentials with subjective probability. Psychophysiology, 14(5), 456-467.

Eimer, M. (2000). Event-related brain potentials distinguish processing stages involved in face perception and recognition. Clinical Neurophysiology, 111(4), 694-705.

Federmeier, K., \& Kutas, M. (2001). Meaning and modality: Influences of context, semantic memory organization, and perceptual predictability on picture processing. Journal of Experimental Psychology: Learning, Memory, and Cognition, 27(1), 202-224.

Fisch, B. (2000). Basic principles of digital and analog EEG. New York, NY: Elsevier Health Sciences. 
Fried-Oken, M., Mooney, A., Peters, B., \& Oken, B. (2013). A clinical screening protocol for the RSVP keyboard brain-computer interface. Disability and Rehabilitation: Assistive Technology, 10(1), 11-18.

Friedrich, E. V., McFarland, D. J., Neuper, C., Vaughan, T. M., Brunner, P., \& Wolpaw, J. R. (2008). A scanning protocol for a sensorimotor rhythm-based brain-computer interface. Biological Psychology, 8o(2), 169-175.

Friedrich, E. V., Scherer, R., \& Neuper, C. (2012). The effect of distinct mental strategies on classification performance for brain-computer interfaces. International Journal of Psychophysiology, 84(1), 86-94.

Geronimo, A. M., Simmons, Z., \& Schiff, S. J. (2016). Performance predictors of brain-computer interfaces in patients with amyotrophic lateral sclerosis. Journal of Neural Engineering, 13(2), 026002.

Guger, C., Daban, S., Sellers, E., Holzner, C., Krausz, G., Carabalona, R., ... Edlinger, G. (2009). How many people are able to control a P30o-based braincomputer interface (BCI)? Neuroscience Letters, 462(1), 94-98.

Hakkarainen, E., Pirilä, S., Kaartinen, J., \& Meere, J. J. (2012). Stimulus evaluation, event preparation, and motor action planning in young patients with mild spastic cerebral palsy: An event-related brain potential study. Journal of Child Neurology, 27(4), 465-470.

Hanagasi, H. A., Gurvit, I. H., Ermutlu, N., Kaptanoglu, G., Karamursel, S., Idrisoglu, H. A., ... Demiralp, T. (2002). Cognitive impairment in amyotrophic lateral sclerosis: Evidence from neuropsychological investigation and eventrelated potentials. Cognitive Brain Research, 14(2), 234-244.

Higger, M., Quivira, F., Akcakaya, M., Moghdamfalahi, M., Nezamfar, H., Cetin, M., \& Erdogmus, M. (2017). Recursive Bayesian coding for BCIs. IEEE Transactions on Neural Systems and Rehabilitation Engineering, 25(6), 704-714.

Hill, N. J., Ricci, E., Haider, S., McCane, L. M., Heckman, S., Wolpaw, J. R., \& Vaughan, T. M. (2014). A practical, intuitive brain-computer interface for communicating 'yes' or 'no' by listening. Journal of Neural Engineering, 11(3), 035003.

Hill, N. J., \& Schölkopf, B. (2012). An online brain-computer interface based on shifting attention to concurrent streams of auditory stimuli. Journal of Neural Engineering, 9(2), 026011.

Horwitz, A., Thomsen, M. D., Wiegand, I., Horwitz, H., Klemp, M., Nikolic, M., ... Benedek, K. (2017). Visual steady state in relation to age and cognitive function. PLOS ONE, 12(2), eo171859.

Hwang, H. J., Han, C. H., Lim, J. H., Kim, Y. W., Choi, S. I., An, K. O., ... Im, C. H. (2017). Clinical feasibility of brain-computer interface based on steady-state visual evoked potential in patients with locked-in syndrome: Case studies. Psychophysiology, 54(3), 444-451.

Hwang, H. J., Lim, J. H., Jung, Y. J., Choi, H., Lee, S. W., \& Im, C. H. (2012). Development of an SSVEP-based BCI spelling system adopting a QWERTY-style LED keyboard. Journal of Neuroscience Methods, 208(1), 59-65. 
Ikegami, S., Takano, K., Saeki, N., \& Kansaku, K. (2011). Operation of a P300based brain-computer interface by individuals with cervical spinal cord injury. Clinical Neurophysiology, 122(5), 991-996.

Jasper, H. (1958). The ten-twenty electrode system of the International Federation. Electroencephalography and Clinical Neurophysiology, 52(3), 3-6.

Jeunet, C., N'Kaoua, B., \& Lotte, F. (2016). Advances in user-training for mentalimagery-based BCI control: Psychological and cognitive factors and their neural correlates. Progress in Brain Research, 228, 223-235.

Jurcak, V., Tsuzuki, D., \& Dan, I. (2007). 10/20, 10/10, and 10/5 systems revisited: Their validity as relative head-surface-based positioning systems. NeuroImage, 34(4), 1600-1611.

Kasahara, T., Terasaki, K., Ogawa, Y., Ushiba, J., Aramaki, H., \& Masakado, Y. (2012). The correlation between motor impairments and event-related desynchronization during motor imagery in ALS patients. BMC Neuroscience, 13, 66 .

Kaufmann, T., Schulz, S. M., Grünzinger, C., \& Kübler, A. (2011). Flashing characters with famous faces improves ERP-based brain-computer interface performance. Journal of Neural Engineering, 8(5), 056016.

Kellicut-Jones, M. R., \& Sellers, E. W. (2018). P30o brain-computer interface: Comparing faces to size matched non-face stimuli. Brain-Computer Interfaces, 5(1), 30-39.

Kim, D. W., Hwang, H. J., Lim, J. H., Leeb, Y. H., Jung, K. Y., \& Im, C. H. (2011). Classification of selective attention to auditory stimuli: Toward vision-free brain-computer interfacing. Journal of Neuroscience Methods, 197, 180-185.

Koch Fager, S., Fried-Oken, M., Jakobs, T., \& Beukelman, D. R. (2019). New and emerging access technologies for adults with complex communication needs and severe motor impairments: State of the science. Augmentative and Alternative Communication, 35, 13-25.

Kübler, A., Furdea, A., Halder, S., Hammer, E. M., Nijboer, F., \& Kotchoubey, B. (2009). A brain-computer interface controlled auditory event-related potential (P300) spelling system for locked-in patients. Annals of the New York Academy of Sciences, 1157(1), 90-100.

Kübler, A., Kotchoubey, B., Hinterberger, T., Ghanayim, N., Perelmouter, J., Schauer, M., ... Birbaumer, N. (1999). The thought translation device: A neurophysiological approach to communication in total motor paralysis. Experimental Brain Research, 124(2), 223-232.

Kübler, A., Nijboer, F., Mellinger, J., Vaughan, T. M., Pawelzik, H., Schalk, G., . . . Wolpaw, J. R. (2005). Patients with ALS can use sensorimotor rhythms to operate a brain-computer interface. Neurology, 64(10), 1775-1777.

Kuhlman, W. N. (1978). Functional topography of the human mu rhythm. Electroencephalography and Clinical Neurophysiology, 44(1), 83-93.

Kutas, M., \& Federmeier, K. D. (2009). N40o. Scholarpedia, 4(10), 7790.

Kutas, M., \& Federmeier, K. D. (2011). Thirty years and counting: Finding meaning the N400 component of the event related brain potential (ERP). Annual Review of Psychology, 62, 621-647. 
Kutas, M., \& Hillyard, S. A. (1980a). Event-related brain potentials to semantically inappropriate and surprisingly large words. Biological Psychology, 11, 99-116.

Kutas, M., \& Hillyard, S. A. (1980b). Reading between the lines: Event-related brain potentials during natural sentence processing. Brain and Language, 11(2), 354-373.

Kutas, M., \& Hillyard, S. A. (1980c). Reading senseless sentences: Brain potentials reflect semantic incongruity. Science, 207, 203-205.

Kutas, M., \& Hillyard, S. A. (1982). The lateral distribution of event-related potentials during sentence processing. Neuropsychologia, 20(5), 579-590.

Kutas, M., \& Hillyard, S. A. (1983). Event-related brain potentials to grammatical errors and semantic anomalies. Memory and Cognition, 11(5), 539-550.

Kutas, M., \& Hillyard, S. A. (1984). Brain potentials reflect word expectancy and semantic association during reading. Nature, 307, 161-163.

Lau, E. F., Phillips, C., \& Poeppel, D. (2008). A cortical network for semantics: (De)constructing the N400. Nature Reviews Neuroscience, 9(12), 920-933.

Liégeois-Chauvel, C., Lorenzi, C., Trébuchon, A., Régis, J., \& Chauvel, P. (2004). Temporal envelope processing in the human left and right auditory cortices. Cerebral Cortex, 14(7), 731-740.

Lin, Z., Zhang, C., Wu, W., \& Gao, X. (2007). Frequency recognition based on canonical correlation analysis for SSVEP-based BCIs. IEEE Transactions on Biomedical Engineering, 54(6), 1172-1176.

Luck, S. (2014). An introduction to the event-related potential technique (2nd ed.). Cambridge, MA: MIT Press.

Lukhanina, E. P., Karaban, I. N., Burenok, Y. A., Mel'nik, N. A., \& Berezetskaya, N. M. (2006). Two phases of the contingent negative variation in humans: Association with motor and mental functions. Neuroscience and Behavioral Physiology, 36(4), 359-365.

Mannarelli, D., Pauletti, C., Locuratolo, N., Vanacore, N., Frasca, V., Trebbastoni, A., ... Fattapposta, F. (2014). Attentional processing in bulbar- and spinalonset amyotrophic lateral sclerosis: Insights from event-related potentials. Amyotrophic Lateral Sclerosis and Frontotemporal Degeneration, 15(1-2), 30-38.

Marchetti, M., \& Priftis, K. (2015). Brain-computer interfaces in amyotrophic lateral sclerosis: A metanalysis. Clinical Neurophysiology, 126(6), 1255-1263.

McCord, M. S., \& Soto, G. (2004). Perceptions of AAC: An ethnographic investigation of Mexican-American families. Augmentative and Alternative Communication, 20(4), 209-227.

McFarland, D. J., Sarnacki, W. A., Townsend, G., Vaughan, T., \& Wolpaw, J. R. (2011). The P30o-based brain-computer interface (BCI): Effects of stimulus rate. Clinical Neurophysiology, 122(4), 731-737.

Mehta, J., \& Jerger, J. (2014). Semantic priming effect across life span: An ERP study. International Journal of Audiology, 53(4), 235-242.

Mehta, J., Jerger, S., Jerger, J., \& Martin, J. (2009). Electrophysiological correlates of word comprehension: ERP and ICA analysis. International Journal of Audiology, 48(1), 1-11. 
Morash, V., Bai, O., Furlani, S., Lin, P., \& Hallett, M. (2008). Classifying EEG signals preceding right hand, left hand, tongue, and right foot movements and motor imageries. Clinical Neurophysiology, 119(11), 2570-2578.

Müller-Putz, G. R., Scherer, R., Brauneis, C., \& Pfurtscheller, G. (2005). Steadystate visual evoked potential (SSVEP)-based communication: Impact of harmonic frequency components. Journal of Neural Engineering, 2(4), 123-130.

Nagai, Y., Critchley, H. D., Featherstone, E., Fenwick, P. B., Trimble, M. R., \& Dolan, R. J. (2004). Brain activity relating to the contingent negative variation: An fMRI investigation. NeuroImage, 21(4), 1232-1241.

Neumann, N., \& Birbaumer, N. (2003). Predictors of successful self control during brain-computer communication. Journal of Neurology, Neurosurgery \& Psychiatry, 74(8), 1117-1121.

Neuper, C., \& Pfurtscheller, G. (2001). Event-related dynamics of cortical rhythms: Frequency-specific features and functional correlates. International Journal of Psychophysiology, 43(1), 41-58.

Neuper, C., Scherer, R., Reiner, M., \& Pfurtscheller, G. (2005). Imagery of motor actions: Differential effects of kinesthetic and visual-motor mode of imagery in single-trial EEG. Cognitive brain research, 25(3), 668-677.

Neville, H., Coffey, S., Holcomb, P., \& Tallal, P. (1993). The neurobiology of sensory and language processing in language impaired children. Journal of Cognitive Neuroscience, 5, 235-253.

Neville, H., Coffey, S., Lawson, D., Fischer, A., Emmorey, K., \& Bellugi, U. (1997). Neural systems mediating American sign language: Effects of sensory experience and age of acquisition. Brain and Language, 57, 285-308.

Nijboer, F., Birbaumer, N., \& Kübler, A. (2010). The influence of psychological state and motivation on brain -computer interface performance in patients with amyotrophic lateral sclerosis-a longitudinal study. Frontiers in Neuroscience, 4(55), 1-13.

Nijboer, F., Furdea, A., Gunst, I., Mellinger, J., McFarland, D. J., Birbaumer, N., \& Kübler, A. (2008). An auditory brain-computer interface (BCI). Journal of Neuroscience Methods, 167(1), 43-50.

Obermaier, B., Muller, G. R., \& Pfurtscheller, G. (2003). "Virtual keyboard" controlled by spontaneous EEG activity. IEEE Transactions on Neural Systems and Rehabilitation Engineering, 11(4), 422-426.

Oken, B. S., Orhan, U., Roark, B., Erdogmus, D., Fowler, A., Mooney, A., . . . Fried-Oken, M. B. (2014). Brain-computer interface with language modelelectroencephalography fusion for locked-in syndrome. Neurorehabilitation and Neural Repair, 28(4), 387-394.

Peters, B., Higger, M., Quivira, F., Bedrick, S., Dudy, S., Eddy, B., . . O Oken, B. (2018). Effects of simulated visual acuity and ocular motility impairments on SSVEP brain-computer interface performance: An experiment with Shuffle Speller. Brain-Computer Interfaces, 5(2-3), 58-72.

Pfurtscheller, G., Brunner, C., Schlögl, A., \& Lopes da Silva, F. H. (2006). Mu rhythm (de) synchronization and EEG single-trial classification of different motor imagery tasks. NeuroImage, 31(1), 153-159. 
Pfurtscheller, G., \& Lopes da Silva, F. H. (1999). Event-related EEG/ MEG synchronization and desynchronization: Basic principles. Clinical Neurophysiology, 110(11), 1842-1857.

Pfurtscheller, G., \& Neuper, C. (1997). Motor imagery activates primary sensorimotor area in humans. Neuroscience Letters, 239(2-3), 65-68.

Pfurtscheller, G., \& Neuper, C. (2010). Dynamics of sensorimotor oscillations in a motor task. In B. Graimann, G. Pfurtscheller, \& B. Allison (Eds.), Braincomputer interfaces. The Frontiers Collection (pp. 47-64). Berlin/Heidelberg, Germany: Springer.

Pitt, K., \& Brumberg, J. (2018a). A screening protocol incorporating braincomputer interface feature matching considerations for augmentative and alternative communication. Assistive Technology, 1-12. https://doi.org/10.1080 $\not 10400435.2018 .1512175$

Pitt, K., \& Brumberg, J. (2018b). Guidelines for feature matching assessment of brain-computer interfaces for augmentative and alternative communication. American Journal of Speech-Language Pathology, 27(3), 950-964.

Pitt, K., Brumberg, J., \& Pitt, A. (2019). Considering augmentative and alternative communication research for brain-computer interface practice. Assistive Technology Outcomes and Benefits, 13, 1-20.

Plante, E., Petten, C. V., \& Senkfor, A. J. (2000). Electrophysiological dissociation between verbal and non-verbal semantic processing in learning disabled adults. Neuropsychologia, 38, 1669-1684.

Polich, J. (2007). Updating P300: An integrative theory of P3a and P3b. Clinical Neurophysiology, 118(10), 2128-2148.

Polich, J., Howard, L., \& Starr, A. (1983). P30o latency correlates with digit span. Psychophysiology, 20(6), 665-669.

Regan, D. (1966). Some characteristics of average steady-state and transient responses evoked by modulated light. Electroencephalography and Clinical Neurophysiology, 20(3), 238-248.

Scherer, R., Billinger, M., Wagner, J., Schwarz, A., Hettich, D. T., Bolinger, E., . . . Müller-Putz, G. (2015). Thought-based row-column scanning communication board for individuals with cerebral palsy. Annals of Physical and Rehabilitation Medicine, 58(1), 14-22.

Segalowitz, S. J., \& Davies, P. L. (2004). Charting the maturation of the frontal lobe: An electrophysiological strategy. Brain and Cognition, 55(1), 116-133.

Sellers, E. W., Krusienski, D. J., McFarland, D. J., Vaughan, T. M., \& Wolpaw, J. R. (2006). A P3oo event-related potential brain-computer interface (BCI): The effects of matrix size and inter stimulus interval on performance. Biological Psychology, 73(3), 242-252.

Srinivasan, R., Bibi, F. A., \& Nunez, P. L. (2006). Steady-state visual evoked potentials: Distributed local sources and wavelike dynamics are sensitive to flicker frequency. Brain Topography, 18(3), 167-187.

Suffcynski, P., Pijn, P. J. M., Pfurtscheller, G., \& Lopes da Silva, F. H. (1999). Event-related dynamics of alpha band rhythms: A neuronal network model of focal ERD/surround ERS. In Event-related desynchronization. Handbook of 
electroencephalography and clinical neurophysiology (Rev. ed., Vol. 6, pp. 6785). Amsterdam, the Netherlands: Elsevier.

Sutton, S., Braren, M., Zubin, J., \& John, E. R. (1965). Evoked-potential correlates of stimulus uncertainty. Science, 150(3700), 1187-1188.

Taylor, B. K., Gavin, W. J., \& Davies, P. L. (2016). The test-retest reliability of the visually evoked contingent negative variation (CNV) in children and adults. Developmental Neuropsychology, 41(3), 162-175.

Teplan, M. (2002). Fundamentals of EEG measurement. Measurement Science Review, 2(2), 1-11.

Van Petten, C., \& Luka, B. J. (2006). Neural localization of semantic context effects in electromagnetic and hemodynamic studies. Brain and Language, 97, 279-293.

Vaughan, T. M., McFarland, D. J., Schalk, G., Sarnacki, W. A., Krusienski, D. J., Sellers, E. W., \& Wolpaw, J. R. (2006). The wadsworth BCI research and development program: At home with BCI. IEEE Transactions on Neural Systems and Rehabilitation Engineering, 14(2), 229-233.

Walter, W. G., Cooper, R., Aldridge, V. J., McCallum, W. C., \& Winter, A. L. (1964). Contingent negative variation: An electric sign of sensori-motor association and expectancy in the human brain. Nature, 203, 380-384.

Wolpaw, J. R., Bedlack, R. S., Reda, D. J., Ringer, R. J., Banks, P. G., Vaughan, T. M., ... Ruff, R. L. (2018). Independent home use of a brain-computer interface by people with amyotrophic lateral sclerosis. Neurology, 91(3), e258-e267.

Wolpaw, J. R., Birbaumer, N., McFarland, D. J., Pfurtscheller, G., \& Vaughan, T. M. (2002). Brain-computer interfaces for communication and control. Clinical Neurophysiology, 113(6), 767-791.

Wolpaw, J. R., \& Boulay, C. B. (2009). Brain signals for brain-computer interfaces. In B. Graimann, G. Pfurtscheller \& B. Allison (Eds.), Brain-computer interfaces (pp. 29-46). Springer/Heidelberg, Germany: Springer.

Wolpaw, J. R., \& McFarland, D. J. (2004). Control of a two-dimensional movement signal by a noninvasive brain-computer interface in humans. Proceedings of the National Academy of Sciences of the United States of America, 101(51), 17849-17854.

Xie, Q., Pan, J., Chen, Y., He, Y., Ni, X., Zhang, J., ... Yu, R. (2018). A gazeindependent audiovisual brain-computer interface for detecting awareness of patients with disorders of consciousness. BMC Neurology, 18(1), 144. 